that some of the cholangiocytes within the ductular reactions costained for Gli2 and either the mesenchymal marker S100A4 or vimentin. It is plausible that EMT occurs in a subset of reactive cholangiocytes within the ductular reaction. As proposed by the authors, ductular cells that respond to hedgehog signaling may represent a hepatic progenitor cell population capable of differentiating into fibrogenic cell types in response to liver injury. Support for this comes from cell culture studies indicating that cholangiocytes are capable of this type of transformation in vitro (6). In addition, Yovchev and colleagues have isolated a subset of hepatic progenitor cells that express both epithelial and mesenchymal markers, including several markers of EMT that are capable of repopulating injured rat liver (13). It would be useful to investigate whether the progenitor cells isolated in their study also respond to hedgehog signaling in a coculture system. Such evidence would provide support for the notion that hedgehog-responsive ductular cells represent a hepatic progenitor cell population. However, definitive proof that cholangiocytes are able to transdifferentiate into myofibroblasts will require in vivo lineage tracing with cholangiocyte-specific marker genes.

We can conclude from this study (12) that hedgehog signaling is likely to medi- ate both beneficial and deleterious effects of liver injury, depending upon the balance between its action as a survival factor for cholangiocytes and as a profibrogenic agent. Further characterization of the mechanisms of hepatic repair regulated by the hedgehog pathway and potential synergistic interaction with other signaling pathways involved in both cholangiocyte proliferation and fibrogenesis will be necessary prior to attempting to enhance or inhibit hedgehog signaling in chronic fibrotic liver diseases.

\section{Acknowledgments}

I thank Rebecca Wells, Klaus H. Kaestner, and Joshua R. Friedman for helpful suggestions on the manuscript.

Address correspondence to: Linda E. Greenbaum, Division of Gastroenterology, Department of Medicine, University of Pennsylvania, 600 Clinical Research Building, 415 Curie Boulevard, Philadelphia, Pennsylvania 19104, USA. Phone: (215) 573-1868; Fax: (215) 573-2024; E-mail: greenbal@mail.med.upenn.edu.

1. Xia, X., et al. 2007. Cholangiocyte injury and ductopenic syndromes. Semin. Liver Dis. 27:401-412.

2. Lazaridis, K.N., Strazzabosco, M., and Larusso, N.F. 2004. The cholangiopathies: disorders of biliary epithelia. Gastroenterology. 127:1565-1577.
3. Lee, J.M., Dedhar, S., Kalluri, R., and Thompson, E.W. 2006. The epithelial-mesenchymal transition: new insights in signaling, development, and disease. J. Cell Biol. 172:973-981.

4. Baum, B., Settleman, J., and Quinlan, M.P. 2008. Transitions between epithelial and mesenchymal states in development and disease. Semin. Cell Dev. Biol. 19:294-308.

5. Robertson, H., Kirby, J.A., Yip, W.W., Jones, D.E., and Burt, A.D. 2007. Biliary epithelial-mesenchymal transition in posttransplantation recurrence of primary biliary cirrhosis. Hepatology. 45:977-981.

6. Rygiel, K.A., et al. 2008. Epithelial-mesenchymal transition contributes to portal tract fibrogenesis during human chronic liver disease. Lab. Invest. 88:112-123.

7. Diaz, R., et al. 2008. Evidence for the epithelial to mesenchymal transition in biliary atresia fibrosis. Hum. Pathol. 39:102-115.

8. Omenetti, A., et al. 2007. Hedgehog-mediated mesenchymal-epithelial interactions modulate hepatic response to bile duct ligation. Lab. Invest. 87:499-514.

9. Omenetti, A., et al. 2008. The hedgehog pathway regulates remodeling responses to biliary obstruction in rats. Gut. 57:1275-1282.

10. Huber, M.A., Kraut, N., and Beug, H. 2005. Molecular requirements for epithelial-mesenchymal transition during tumor progression. Curr. Opin. Cell Biol. 17:548-558.

11. Li, X., Deng, W., Lobo-Ruppert, S.M., and Ruppert, J.M. 2007. Gli1 acts through Snail and E-cadherin to promote nuclear signaling by beta-catenin. Oncogene. 26:4489-4498.

12. Omenetti, A., et al. 2008. Hedgehog signaling regulates epithelial-mesenchymal transition during biliary fibrosis in rodents and humans. J. Clin. Invest. 118:3331-3342.

13. Yovchev, M.I., et al. 2008. Identification of adult hepatic progenitor cells capable of repopulating injured rat liver. Hepatology. 47:636-647.

\title{
Prenatal maternal diet affects asthma risk in offspring
}

\author{
Rachel L. Miller \\ Division of Pulmonary, Allergy, and Critical Care Medicine, Columbia University College of Physicians and Surgeons, New York, New York, USA.
}

\begin{abstract}
Recently, epigenetic-mediated mechanisms - which involve heritable changes in gene expression in the absence of alterations in DNA sequences - have been proposed as contributing to asthma. In this issue of the JCI, Hollingsworth and colleagues report on the effect of prenatal maternal dietary intake of methyl donors on the risk of allergic airway disease in offspring in mice and show that these effects involve epigenetic regulation (see the related article beginning on page 3462). Supplementation of the maternal diet with methyl donors was associated with greater airway allergic inflammation and IgE production in F1 and, to some extent, F2 progeny. Site-specific differences in DNA methylation and reduced transcriptional activity were detected. If these findings are confirmed, a new paradigm for asthma pathogenesis may be emerging.
\end{abstract}

Conflict of interest: The author has declared that no conflict of interest exists.

Citation for this article: J. Clin. Invest. 118:3265-3268 (2008). doi:10.1172/JCI37171.
More and more, it seems that our traditional view of asthma as a complex disease that is mediated by a genetic predisposition and childhood or later environmental exposures needs updating. At this point, a mounting body of literature has established that prenatal exposures can influence the risk for developing asthma (1). This link has been most firmly documented in epidemiological studies of prenatal exposure to cigarette smoke and subsequent wheeze. For example, in a large prospective Danish cohort study of over 11,000 children, maternal smoking at the 36th week of gestation was associated with transient wheezing in children before age 3 years (2). In a Stockholm cohort of over 4,000 newborns, maternal smoking during but not following pregnancy was associated with an increased risk of recurrent wheezing in offspring up to age two years (3). In mouse 


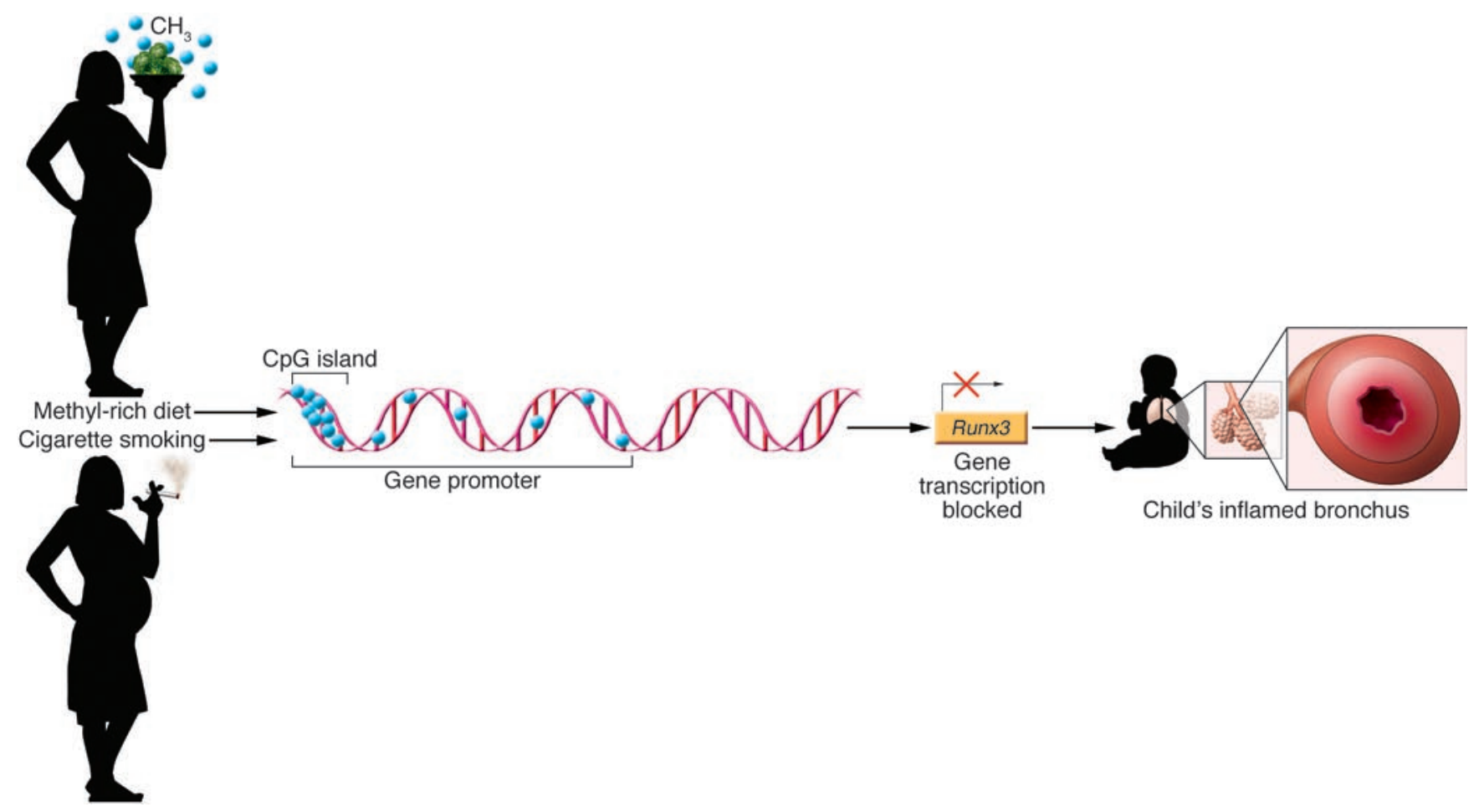

Figure 1

Prenatal maternal diet affects asthma risk in offspring. Prenatal maternal environmental exposure to diets high in folate, vitamin $\mathrm{B}_{12}$, choline, and methionine, which provide methyl donors, as well as to cigarette smoking may be associated with the covalent addition of a methyl group to cytosines in $\mathrm{CpG}$ dinucleotides and other epigenetic changes. These changes in turn may repress gene transcription and induce asthma phenotypes (i.e., airway allergic inflammation) in the offspring. As reported by Hollingsworth and colleagues in their study in mice in this issue of the $\mathrm{JCl}$ (25), prenatal methyl-rich diets may promote DNA methylation and reduce transcription of genes associated with the downregulation of allergic immune responses in the airway, such as runt-related transcription factor 3 (Runx3).

models, exposure during pregnancy to an air pollutant aerosol (residual oil fly ash) led to a greater susceptibility to an asthmalike phenotype in the offspring mice (4).

\section{Prenatal diet and atopy risk}

The relationship between a mother's diet during pregnancy and the child's subsequent risk of developing asthma or atopy has become a topic of growing investigation. Reduced maternal intake of vitamin E, vitamin $\mathrm{D}$, and zinc during pregnancy all have been associated with a greater risk of development of asthma and wheezing symptoms in 5-year-old children $(5,6)$. Most recently, Chatzi and colleagues found that adherence to a Mediterranean diet during pregnancy was associated with protection from persistent wheeze and atopy in children (7). Frequent maternal intake of fish during pregnancy also reduced the risk of food and possibly inhalant allergic sensitizations (8). Daily consumption of nut products during pregnancy has been associated with asthma in the child by age 8 years (9). However, in a recent meta- analysis of four clinical studies, avoidance of specific food antigens during pregnancy did not appear to influence the risk of development of atopic disease in the child (10). Combined, these studies suggest that the prenatal diet can alter the intrauterine environment in complex and possibly inconsistent ways.

\section{So how does this happen? Epigenetic regulation}

Current hypotheses tend to consider either immune-mediated or epigenetic-mediated mechanisms. It now appears evident that antigen-specific B cell and $T$ cell immune responses can develop following antigen exposures during pregnancy, as our group has demonstrated following prenatal vaccination against influenza antigens (11). Prenatal environmental exposures may be able to alter the intrauterine cytokine milieu (12). However, proof that prenatal dietary exposure delays or prevents early sensitization to food antigens and hence risk for clinical atopy has so far been more elusive (13). Lately, epigenetic-mediated mechanisms that entail heritable changes in gene expression that occur in the absence of alterations in DNA sequences have been proposed (14). These changes usually involve either DNA methylation (covalent addition of a methyl group to cytosines in CpG dinucleotides) or chromatin packaging of DNA via posttranslational modifications of histones (Figure 1).

In their seminal studies, Cooney and colleagues and Waterland and Jirtle demonstrated that methyl supplementation of the maternal diet during pregnancy with folic acid, vitamin $B_{12}$, and other agents increased DNA methylation of the long terminal repeat controlling the expression of the agouti gene and thereby influenced the methylation-dependent phenotype (coat color distribution) in mouse offspring (15, 16). Maternal stress and impaired nurturing in addition to environmental exposures to xenobiotic chemicals, endocrine disrupters, and low-dose radiation all have been associated with epigenetic gene regulation without changing the DNA sequence (reviewed in refs. 17, 18). 
Epigenetic regulation also may be inherited across multiple generations, implying that not only prenatal maternal but also grandmaternal exposures may influence subsequent gene expression in the children. Examples of multigenerational effects on phenotype have been demonstrated in studies on the kinked tail (Axin $\left.{ }^{\mathrm{Fu}}\right)$ and agouti-viable yellow ( $\mathrm{A}^{\mathrm{vy}}$ ) allele, associations between endocrine disrupters and male infertility, and hormone-dependent cancer risk (18). While clearly multigenerational effects of prenatal environmental exposures are difficult to demonstrate in human epidemiological studies, one study is quite suggestive. In a provocative nested case control study by the Children's Health Study of children diagnosed with asthma by age 5 years versus control subjects, grandmaternal smoking during pregnancy was associated with a greater risk for developing asthma in grandchildren. Borderline statistical significance was achieved when the effect was studied independently of maternal smoking (19).

In the asthma literature, articles supporting epigenetic mechanisms are just beginning to be published. These include mouse studies demonstrating that inhaled diesel exposure and intranasal Aspergillus fumigatus allergen induced hypermethylation at multiple sites of the IFN- $\gamma$ promoter and hypomethylation at the CpG-408 site of the IL-4 promoter. Altered methylation of both gene promoters was correlated significantly with changes in IgE levels (20). In other recent work, cell-specific DNA methylation at the A disintegrin and metalloprotease 33 (ADAM33) gene promoter, an area whose expression has been implicated in severe asthma, has been reported. In this study, promoter methylation levels differed considerably between epithelial cells and fibroblasts, and this in turn differentially regulated ADAM33 gene expression (21). In another study, bronchial biopsies obtained from untreated asthmatics possessed greater levels of histone acetyltransferase and lower levels of histone deacetylase (HDAC) enzymatic activity. Levels reversed following treatment with inhaled steroids (22). Because, in general, acetylation of histones is associated with gene induction and deacetylation is associated with gene silencing (23), the study suggests that one antiinflammatory mode of action in the pharmacological treatment of asthma may be epigenetic. Finally, trichostatin A-induced inhibition of endogenous HDAC upregulated Th2 cytokine (IL-13,
IL-5) and GATA3-mediated CD45RO ${ }^{+}$ $T$ cell recall responses (24), suggesting that Th polarization can be directly epigenetically regulated.

\section{High-methyl donor prenatal diet and allergic inflammation in offspring}

The study by Hollingsworth and colleagues in this issue of the JCI (25) is the first to report on the effect of dietary methyl donors on the risk for allergic airway disease via epigenetic mechanisms. Using an approach similar to that developed by Waterland and Jirtle when they examined agouti coat color, Hollingsworth's group fed mice a diet supplemented with methyl donors during gestation and weaning. Supplementation of the maternal diet with methyl donors was associated with greater levels of airway hyperactivity, airway eosinophilic inflammation, chemokines $\mathrm{KC}$, macrophage inflammatory protein $1 \beta$ (MIP1 $\beta$ ), and RANTES, and IgE production in the F1 progeny. There was a less robust effect on airway eosinophilic inflammation and IgE level in the F2 generation mice. Furthermore, the authors profiled site-specific differences in DNA methylation in phenotypic extremes of the F1 progeny that were gestated on either a high- or lowmethylation diet. Identifying and focusing on 5 candidate genes in validation geneexpression experiments, they were able to confirm that in utero supplementation with a methyl-rich diet was associated with decreased transcriptional activity and mRNA expression in lung tissue. One of these genes was runt-related transcription factor 3 (Runx3), a gene known to downregulate allergic airway inflammation. Diets supplemented with methyl donors during either lactation or adulthood did not significantly affect the airway disease phenotype of mice, suggesting that the critical time window of susceptibility is gestation. Hence, the authors demonstrated that prenatal diet and methyl donation are associated with altered asthma-related phenotypes across multiple generations via epigenetic mechanisms and also proposed candidate genes and maybe an epigenome that could be (and would need to be) validated in future studies.

\section{Public health implications}

One cannot ignore the observation that the increase in asthma prevalence over recent decades approximately coincides with worldwide campaigns that recommend periconceptional dietary folate supplemen- tation (26). From a public health perspective, the adverse nonrespiratory health consequences of insufficient prenatal folate consumption are legitimate concerns. But an even broader public health issue has surfaced. If confirmed, prenatal exposures may influence the development of asthma not only for our children, but for their children as well.

Despite the provocative nature of the findings of the study by Hollingsworth and colleagues (25), they need to be interpreted with serious caution. Mouse models for asthma, being mouse models, are limited in their direct applicability to human asthma. Translation to the clinical arena would require cohort-driven epigenetic research, particularly from studies powered sufficiently to examine questions of dose and timing of prenatal environmental and/or dietary exposure, on the subsequent clinical risk for asthma. Nonetheless, this study seems to have uncovered a new form of environmental exposure - prenatal maternal dietary exposure to methyl groups - that together with certain genetic events may increase the risk of asthma in offspring. Let us hope that our scientific community can design studies to validate these findings in human disease, not only for the benefit of our children, but potentially for their children as well.

\section{Acknowledgments}

The author is supported by grants R21 ES013063, R01 ES013163, and P50ES015905 from the NIH.

Address correspondence to: Rachel. L. Miller, Division of Pulmonary, Allergy, and Critical Care Medicine, Columbia University College of Physicians and Surgeons, PH8E, 630 W. 168th Street, New York, New York 10032, USA. Phone: (212) 3057759; Fax: (212) 305-2277; E-mail: rlm14@ columbia.edu.

\footnotetext{
1. Herz, U., et al. 2000. Prenatal sensitization in a mouse model. Am. J. Respir. Crit. Care Med. 162:S62-S65.

2. Magnusson, L., Olesen, A., Wennborg, H., and Olsen, J. 2005. Wheezing, asthma, hayfever, and atopic eczema in childhood following exposure to tobacco smoke in fetal life. Clin. Exp. Allergy. 35:1550-1556.

3. Lannero, E., Wickman, M., Pershagen, G., and Nordvall, L. 2006. Maternal smoking during pregnancy increases the risk of recurrent wheezing during the first years of life. Respir. Res. 7:3.

4. Hamada, K., et al. 2007. Exposure of pregnant mice to an air pollutant aerosol increases asthma susceptibility in offspring. J. Toxicol. Environ. Health A. 70:688-695.

5. Devereux, G., et al. 2006. Low maternal vitamin E intake during pregnancy is associated with asthma
} 
in 5-year-old children. Am. J. Respir. Crit. Care Med. 174:499-507.

6. Devereux, G., et al. 2007. Maternal vitamin D intake during pregnancy and early childhood wheezing. Am. J. Clin. Nutr. 85:853-859.

7. Chatzi, L., et al. 2008. Mediterranean diet in pregnancy is protective for wheeze and atopy in childhood. Thorax. 63:507-513

8. Calvani, M., et al. 2006. Consumption of fish, butter and margarine during pregnancy and development of allergic sensitizations in the offspring: role of maternal atopy. Pediatr. Allergy Immunol. 17:94-102.

9. Willers, S.M., et al. 2008. Maternal food consumption during pregnancy and the longitudinal development of childhood asthma. Am. J. Respir. Crit. Care Med. 178:124-131.

10. Kramer, M.S., and Kakuma, R. 2006. Maternal dietary antigen avoidance during pregnancy or lactation, or both, for preventing or treating atopic disease in the child. Cochrane Database Syst. Rev. 3:CD000133.

11. Rastogi, D., et al. 2007. Antigen-specific immune responses to influenza vaccine in utero. J. Clin. Invest. 117:1637-1646.

12. Chung, E.K., Miller, R.L., Wilson, M.T., McGeady, S.J., and Culhane, J.F. 2007. Antenatal risk factors, cytokines and the development of atopic disease in early childhood. Arch. Dis. Child Fetal Neonatal Ed. 92:F68-F73.

13. Sicherer, S.H., and Burks, A.W. 2008. Maternal and infant diets for prevention of allergic diseases: understanding menu changes in 2008. J. Allergy Clin. Immunol. 122:29-33.

14. Miller, R.L., and Ho, S. 2008. Environmental epigenetics and asthma: Current concepts and call for studies. Am. J. Respir. Crit. Care Med. 177:567-573.

15. Cooney, C.A., Dave, A.A., and Wolff, G.L. 2002. Maternal methyl supplements in mice affect epigenetic variation and DNA methylation of offspring. J. Nutr. 132:2393S-2400S.

16. Waterland, R.A., and Jirtle, R.L. 2003. Transposable elements: Targets for early nutritional effects on epigenetic gene regulation. Mol. Cell. Biol. 23:5293-5300.

17. Dolinoy, D., Weidman, J., and Jirtle, R. 2007. Epigenetic gene regulation: linking early developmental environment to adult disease. Reprod. Toxicol. 23:297-307.

18. Tang, W., and Ho, S.M. 2007. Epigenetic reprogramming and imprinting in origins of disease. Rev. Endocr. Metab. Disord. 8:173-182

19. Li, Y.-F., Langholz, B., Salam, M.T., and Gilliland, F.D. 2005. Maternal and grandmaternal smoking patterns are associated with early childhood asthma. Chest. 127:1232-1241.

20. Liu, J., et al. 2008. Combined inhaled diesel exhaust particles and allergen exposure alter methylation of $\mathrm{T}$ helper genes and IgE production in vivo. Toxicol. Sci. 102:76-81.

21. Yang, Y., et al. 2008. Epigenetic mechanisms silence a disintegrin and metalloprotease 33 expression in bronchial epithelial cells. J. Allergy Clin. Immunol. 121:1393-1399.

22. Ito, K., et al. 2002. Expression and activity of histone deacetylases in human asthmatic airway. Am. J. Respir. Crit. Care Med. 166:392-396.

23. Bhavsar, P., Ahmad, T., and Adcock, I. 2008. The role of histone deacetylases in asthma and allergic diseases. J. Allergy Clin. Immunol. 121:580-584.

24. Su, R., Becker, A., Kozyrskyj, A., and Hayglass, K. 2008. Epigenetic regulation of established human type 1 versus type 2 cytokine responses. J. Allergy Clin. Immunol. 121:57-63.

25. Hollingsworth, J.W., et al. 2008. In utero supplementation with methyl donors enhances allergic airway disease in mice. J. Clin. Invest. 118:3462-3469.

26. Eichholze, M., Tönz, O., and Zimmermann, R. 2006. Folic acid: a public-health challenge. Lancet. 367:1352-1361.

\title{
Novel epitope begets a novel pathway in type 1 diabetes progression
}

\author{
Jeffrey A. Frelinger \\ Department of Microbiology and Immunology, University of North Carolina, Chapel Hill, North Carolina, USA.
}

\begin{abstract}
While $\mathrm{CD8}^{+} \mathrm{T}$ cells are critical to diabetogenesis in NOD mice, evidence of their involvement in human type 1 diabetes (T1D) has been circumstantial. The existence of $\mathrm{CD8}^{+} \mathrm{T}$ cells specific for $\beta$ cell peptides has been demonstrated, but functional data regarding the role of these cells in T1D have been lacking. In this issue of the JCI, Skowera et al. describe an unusual selfpeptide epitope derived from the leader sequence of preproinsulin (PPI) and show that $50 \%$ of $\mathrm{HLA}^{-\mathrm{A}^{+}}$patients with new-onset T1D possessed circulating $\mathrm{CD8}^{+} \mathrm{T}$ cells specific for this epitope, suggesting that PPI plays a critical role in the development of T1D (see the related article beginning on page 3390). They also report that $\beta$ cells upregulate PPI expression in the presence of high glucose levels, rendering these cells more susceptible to lysis and potentially accelerating disease. This suggests that interventions aimed at decreasing the PPI-specific $\mathrm{CD}^{+} \mathrm{T}$ cell response early after T1D diagnosis may be efficacious in ameliorating the disease process.
\end{abstract}

Type 1 diabetes (T1D) is an autoimmune disease that results from $\mathrm{T}$ cell-mediated destruction of the $\beta$ cells of the endocrine pancreas. Much of our knowledge about the autoimmune pathogenesis of T1D, including the role of both $\mathrm{CD}^{+}$and $\mathrm{CD}^{+}$

Nonstandard abbreviations used: PPI, preproinsulin; $\mathrm{TAP}$, transporter associated with antigen processing; T1D, type 1 diabetes.

Conflict of interest: The author has declared that no conflict of interest exists.

Citation for this article: J. Clin. Invest. 118:3268-3271 (2008). doi:10.1172/JCI37125.
$\mathrm{T}$ cells in $\beta$ cell destruction, is based on studies of the NOD mouse (1). In both mice and humans, development of T1D requires the interaction of a genetically susceptible host and an environmental insult. Investigation of the genetic basis of T1D susceptibility in NOD mice and comparison of the results with genome scans in humans with T1D have confirmed that many of the same processes are involved in this disease in both species (2). Although more than 20 loci have been described, the major genes that govern T1D susceptibility in both mice and humans map in the major histocompatibility complex. Specific MHC class II alleles in both mice and humans have a strong association with T1D susceptibility. The genetic evidence for MHC class I involvement is less clear. Removal of $\mathrm{CD}^{+} \mathrm{T}$ cells from young NOD mice results in no progression to diabetes (3). Similarly, depletion of $\mathrm{CD}^{+}{ }^{+} \mathrm{T}$ cells from NOD mice also results in the failure to progress to diabetes (4). Adoptive transfer experiments with both purified $\mathrm{T}$ cells from diabetic mice and diabetogenic $T$ cell clones established unequivocally that $\mathrm{CD} 8^{+} \mathrm{T}$ cells are critical for the development of diabetes (5). As a result, there is widespread belief that $\beta$ cell-specific immune responses mediated by both $\mathrm{CD}^{+}$and $\mathrm{CD}^{+} \mathrm{T}$ cells are required for the development of T1D in humans as well as in NOD mice. However, the evidence that $\mathrm{CD}^{+} \mathrm{T}$ cells are critical to the development of T1D in humans remains circumstantial. While $\mathrm{CD}^{+} \mathrm{T}$ cell $\beta$ cell-specific epitopes, restricted by MHC class I, have been detected, the frequency of these epitope-specific $\mathrm{CD} 8^{+} \mathrm{T}$ cell clones is low, and their in vivo function has been difficult to demonstrate (6). 